\title{
DETECTION AND ANALYSIS OF LED DISPLAY SYSTEM IN LARGE STADIUMS
}

\author{
Xiaodong YI and Min ZHOU* \\ School of Physical Education, Sichuan University, Chengdu, Sichuan, 610065, China; \\ *E-mail:dhxutxo4636@126.com
}

\begin{abstract}
As the mainstream products of flat panel display, LED screen is becoming more and more popular, which has been widely used in the display system of large gymnasium. The detection of LED display system in large stadium is analyzed. Based on the large LED display in Jingzhou stadium, the qualification of LED display system after installation is tested. A scanning line seed filling algorithm is proposed and used to collect the gray value in the connected domain of the lamp and obtain the average gray value of the light point, so that the LED display system of the large gymnasium is detected. The experimental results show that a reasonable LED display and adjustment scheme can ensure the installation of LED display system in large gymnasium in line with the requirements of the national standard.
\end{abstract}

Keywords: stadiums and gymnasiums, LED, system testing, OSTU algorithm

\section{INTRODUCTION}

LED display is the latest information media tool. It has the following advantages: long life cycle, less energy consumption, large visibility range, long viewing distance, etc. [1]. At present, it has a wide range of use, for example, securities trading, finance, stations, stadiums, road traffic, airport flights, dispatching command centres, and shopping malls, advertising media and other public places. Compared with LCD, this LED display has the characteristics of low price and high brightness.
And standard cell boards can be used to assemble the size of their own requirements [2]. Lighting is a key component in the construction of sports venues. The design must meet the specific needs of the venue and provide lighting conditions for recreational activities, competition activities, training activities and cleaning activities. Whether the lighting effect is good or not is directly related to the fairness of the game, the effect of broadcasting, the safety of the players and the atmosphere of the scene. However, for large scale gymnasiums, the detection of LED display system is also crucial [3]. The detection of the display system plays a very important role in the lighting. The design of the horse track for controlling the lighting equipment is closely related to the specialties of architecture, structure and electricity [4]. Because it is necessary to examine the LED display system in the design and construction of sports venues, it is necessary to understand the core technology and create a scientific display and detection system to help promote the intelligent development of the gymnasium [5]. Therefore, the detection of large LED display has a very important practical value.

\section{STATE OF THE ART}

In the LED production company of China, when the display system is tested, the LED display unit is opened to use the human eye to check it. However, this kind of manual detection relies on the subjectivity of the individual, and it is difficult to ensure the credibility of the test. On the other hand, the human eye may cause harmful damage to the high bright- 
ness LED display panel [6]. In the west, the development of test facilities for display screen lighting units is early. However, the test target is not uniform and the facilities are expensive. For example, Japanese enterprises are testing the luminous effect of light-emitting devices. This is to ensure the unity of production and invest tens of millions of dollars to develop an automatic inspection, acquisition, correction and production line [7].

In China, Shenzhen elephant horizon Photoelectric Technology Co., Ltd. invested about six hundred thousand in developing a set of module detection system. However, its detection efficiency is not high, and is limited to internal applications only. Other manufacturers are slow in testing, but only for internal use. The rest of the manufacturers did not invest in the development of the display module's testing facilities. In September 3, 1997, China's Ministry of Electronics Industry issued the general specification for display, and has begun to practice. In May 1998, the relevant commission commissioned the Nanjing Luo Pu company to formulate the "screen display test method", which is finalized to promote the steady development of the industry [8]. The LED display test method divides the performance of the LED display, such as light and power, electricity and so on. The methods of testing are categorized. However, this method is clear after the module is assembled into LED display screen. However, the way of testing is not clearly defined. If the detection of LED display module cannot be standardized, it will be harmful to the detection of LED display unit. After the screen, the effect will also be adversely affected.

\section{METHODOLOGY}

\subsection{Image Denoising Algorithm Based on LED Display System Detection}

In the process of collecting, acquiring and transmitting images of a unit panel of LED display, the influence of the input conversion device and the surrounding environment is input. For example, the fluctuation of light intensity, the unevenness of the sensitivity of the photosensitive element, the quantization noise generated during the digitization process, the camera shake fixed on the bracket caused by the machine vibration, the fluctuation of the power supply, the shot noise introduced by the CCD device itself, etc. This makes it inevitable that the digital images acquired in the detection system contain a wide variety of noises and distortions. These noises will bring many difficulties to image processing. It has a direct impact on image segmentation, feature extraction, image recognition and other image processing operations. Therefore, real-time collection of images needs to be filtered first.

In a large number of experiments, it is found that the image captured by the camera is seriously affected by discrete impulse noise and discrete salt and pepper noise. Median filtering is a nonlinear signal processing technique that can effectively suppress salt and pepper noise. The basic principle of median filtering is to replace the gray value of a point in a digital image or a digital sequence with the median value of a point in a neighbourhood. The surrounding pixels are closer to the true value, thus eliminating the isolated noise points. The method is to use a two-dimensional sliding template with some structure to sort the pixels in the template according to the size of pixels. Two dimensional data sequences are generated monotonically (or descending). The two-dimensional median filter output is:

$$
g(x, y)=\operatorname{med}\{f(x-k, y-l),(k, l) \in w\}
$$

Among them, $f(x, y)$ and $g(x, y)$ are original and processed images respectively. $w$ is a two-dimensional template. Usually $2 * 2,3 * 3$ area, generally used window. Median filtering can preserve the details of the image while removing noise. In addition, the median filter is easy to self adapt, so that its filtering performance can be further improved. Therefore, the median filtering method is adopted to remove noise, and the width of the filter window is 3 * 3 area.

\subsection{OSTU Algorithm Based on Image Segmentation}

Image segmentation is one of the key technologies in digital image processing and computer vision. The purpose is to separate the target from the background and provide a basis for subsequent classification and recognition. The image segmentation methods are divided into the following categories: segmentation methods based on threshold include region based segmentation, edge based segmentation, and segmentation based on specific theories. 
Threshold segmentation is one of the most commonly used image segmentation methods. Scholars at home and abroad have done a lot of research and put forward a variety of methods to select ICJ values. Among these methods, the early OSTU method proposed by Otsu is most concerned. It uses an exhaustive search method to select a gray threshold so that the variance between the background and the target is the maximum. Later, Reddi and Morii did not use the original exhaustive search method, and a fast search iteration method was proposed. Because of the low signal-to-noise ratio (SNR) of image, the segmentation results of OSTU algorithm are not very satisfactory. In order to solve the above problem, Liu Jianzhuang uses the idea of two-dimensional histogram proposed by Abutaleb to select the closed value by an average gray level two-dimensional histogram, so that the segmentation effect is obviously improved. But the system needs real-time processing on the one hand, and it can directly observe the large SNR of the picture in the system. Therefore, the system adopts one-dimensional OSTU algorithm for image segmentation. The segmentation principle of the next one dimensional OSTU algorithm is first introduced. For the $\mathrm{M} * \mathrm{~N}$ size gray image, $f(x, y)$ is the gray value of the image at the $(x, y)$ point and the gray level is $L$. $p(k)$ is the frequency of the gray value of $k$, and then there is:

$$
p(k)=\frac{1}{M \times N} \sum_{f(i, j)=k} 1 .
$$

Assuming that the background and target of the image segmented by the gray value $T$ are $\{f(i, j) \leq t\} \mid\{f(i, j)>t\}$, the proportion of the background part is:

$$
\omega_{0}(t)=\sum_{0 \leq i \leq t} p(i)
$$

The proportion of the target part:

$$
\omega_{1}(t)=\sum_{t \leq i \leq L-1} p(i) .
$$

The number of points in the target part:

$$
N_{1}(t)=M N \sum_{t \leq i \leq L-1} p(i) .
$$

Background mean:

$$
\mu_{0}(t)=\sum_{0 \leq i \leq t} \frac{p(i)}{\omega_{0}(t)}
$$

Target mean:

$$
\mu_{1}(t)=\sum_{t \leq i \leq L-1} i \frac{p(i)}{\omega_{1}(t)} .
$$

Total mean:

$$
\mu=\omega_{0}(t) \mu_{0}(t)+\omega_{1}(t) \mu_{1}(t)
$$

The formula for finding the best threshold by using the largest class variance is as follows:

$$
\begin{gathered}
g=\arg \max _{0 \leq t \leq L-1}\left\{\omega_{0}(t)\left[\mu_{0}(t)-\mu^{2}\right]+\right. \\
\left.+\omega_{1}(t)\left[\mu_{1}(t)-\mu\right]^{2}\right\} .
\end{gathered}
$$

After the partition threshold is determined, the definition is:

$$
f(x, y)=\left\{\begin{array}{l}
0 ; f(x, y)<g \\
255 ; \text { other }
\end{array} .\right.
$$

Thus the image segmentation is completed. As shown in Fig.1, the result of threshold segmentation for indoor model is calculated by the above method, and the best segmentation threshold is gray level.

Since the glass mirror surface of the display panel will have some particles and the glow of the display unit after the light is lit, the image edge of the two valued image will be scattered with some noise areas, such as the sharp corner of the edge of the lamp area in the lower image. The elimination of these noises is based on the introduction of morphological image processing to handle the edges of these regions. There are four basic operations in morphology: dilation, decay, candlestick opening and closing operations. When $A$ and $B$ are collections of $Z 2, A$ is defined as $A \oplus B$ by $B$ expansion:

$$
A \oplus B=\left\{Z \mid(\hat{B})_{Z} \cap A \neq \otimes\right\} .
$$

This formula is based on the mapping of the $B$ relative to its origin and the displacement of the image by $Z$. A is expanded by $B$ as a set of all displacement $Z$, so that at least one element of $\hat{B}$ and $A$ is overlapped. The use of pairs of rotting candles is the collection of points contained in all translations. One of the simplest uses of rotting candles is to eliminate irrelevant details from two valued images. Dilation and rotten worms are mutually ex- 


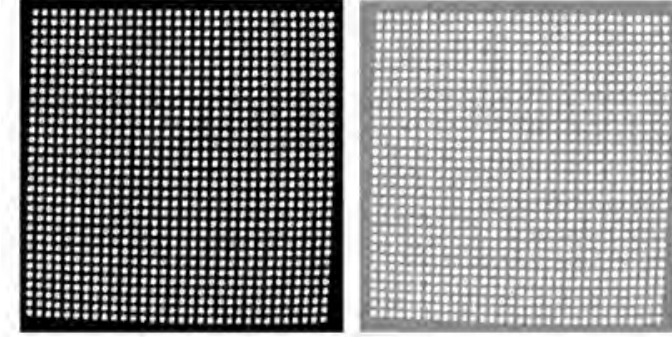

Fig.1. Images before and after threshold method segmentation

clusive for set complement operation and reflection operation.

\subsection{Experimental Setup}

The planning site for Jingzhou cultural and sports centre is 29.6 hectares, with a total construction area of about 33 thousand and 600 square meters. The LED large screen of the Main Gymnasium of Sports Centre is adopt two P7.62 indoor full colour LED displays, each display area is $93.352 \mathrm{~m}^{2}$. The display screen is installed on the wall of the main hall's 5-J axis and the 15-J shaft respectively, which is installed by the embedded structure of the box body. The swimming pool uses a P7.62 indoor full colour LED display with an area of $30.482 \mathrm{~m}^{2}$. The display screen is installed on the wall of the 1-F axis of the swimming pool, which is also installed with an embedded structure of the box.

The LED display system is located in the gymnasium of Jingzhou sports centre. The long axis of the site is north-south, with a fixed stand around it, and the rostrum is on the west side. A total of two LED display screens are installed on the venue, which are installed on the north and south ends of the audience auditorium, respectively, with three primary colours (full colour) LED display. According to the methods stipulated by the GB/T 294582012 (sports venues LED display requirements and test methods) standard, the brightness uniformity of the two sets of the three base colour LED displays of the stadiums and gymnasiums is measured respectively. The average temperature of the field environment is $29.40 \mathrm{C}$, and the relative humidity is $76 \%$. LED full colour screen basic information is shown in Table 1.

According to the size of the display screen and the location of the display screen, the monitoring points of the display screen are set up. According to the size of the actual display screen, in or-
Table 1. LED Full Colour Screen Basic Information

\begin{tabular}{|c|c|c|}
\hline \multirow{4}{*}{ Size } & Length & $13664 \mathrm{~mm}$ \\
\cline { 2 - 3 } & Width & $6832 \mathrm{~mm}$ \\
\hline \multirow{4}{*}{$\begin{array}{c}\text { Full colour } \\
\text { screen (two } \\
\text { sets) }\end{array}$} & type & $\begin{array}{c}\text { P7.62 } \\
7.62 \mathrm{~mm} \text { LED }\end{array}$ \\
\cline { 2 - 3 } & Pixel spacing & $7.62 \mathrm{~mm}$ \\
\cline { 2 - 3 } & Paintenance & $\begin{array}{c}\text { Backstage } \\
\text { maintenance }\end{array}$ \\
\cline { 2 - 3 } & Installation & Wall hanging \\
\cline { 2 - 3 } & method & $* * *$ \\
\cline { 2 - 3 } & Supplier & point $/ \mathrm{m}^{2}$ \\
\hline
\end{tabular}

der to ensure the rationality of the display system detection, the display area is divided into nine areas. And the monitoring points are set up. The maximum brightness of LED display is a basic rigid index in LED detection project. In engineering projects, first of all, luminance should meet the contract requirements. At the same time, many screen manufacturers in China are also concerned about this project goal. In the process of luminance detection, the key to luminance detection is to find the optical axis of the display screen. Because of the huge display screen of Jingzhou Sports Centre, in order to ensure a good visual effect for the audience in the field, the LED display panel with high hanging height is usually installed with a certain degree of tilt in addition to a certain height. So, finding the optical axis is the first task to test the brightness. On the other hand, it is necessary to ensure that the display screen is in a reasonable operating time range when testing the brightness of the display. Try to avoid the brightness attenuation and surface area caused by LED long time use, resulting in a decrease in brightness of the display screen.

Homogeneity refers to the combination of the intensity uniformity of the pixel, the brightness uniformity of the display module and the brightness uniformity of the module in the display screen. These three indicators play a great role in the production inspection of manufacturers. Due to the influence of environmental factors on the installation of LED display screen, the difficulty of detecting the uniformity of the whole screen is greatly increased. It is mainly because of the difficulty in measuring the light intensity of the outdoor pixels 


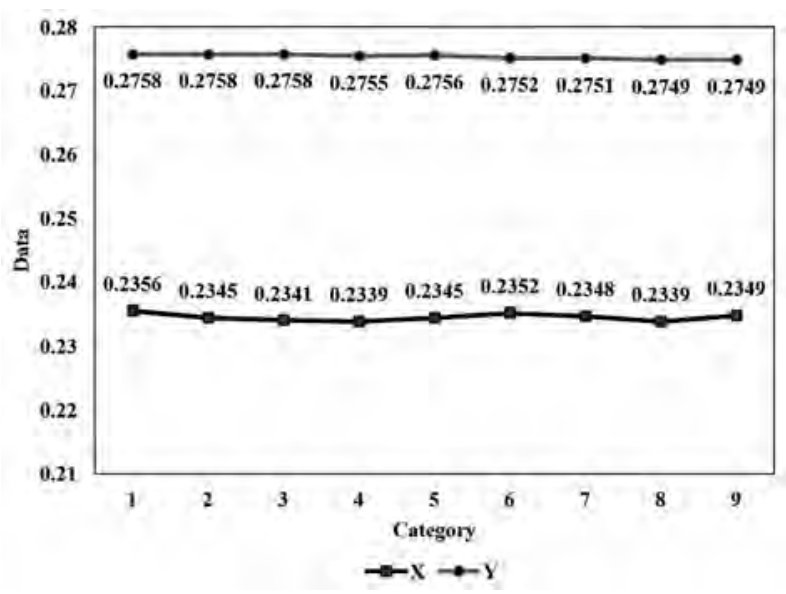

Fig.2. Results of colour coordinate detection in white field

and the confusion of the installation definition of the display module. So the field detection is only pertinent, and it does not have the actual significance of the detection.

Contrast is an optical performance indicator of LED display detection, which mainly reflects the brightness of the display, and can also reflect the influence of the environment on the brightness of the display screen. Therefore, when measuring the brightness contrast of LED display, it is necessary to measure according to the different contents of the scene, and set up different scenes for measuring.

\section{RESULT ANALYSIS AND DISCUSSION}

With the improvement of the LED display control technology, the display can adjust every colour of the display in the process of use, so that the display screen is displayed in a smooth and perfect condition. According to the LED display test method, its detection technology is the white field colour of the highest gray level and the most luminance display, reflecting the brightness of the R G and the brightness of the three colours in the brightest spot. The average value of $x$ coordinates of the test points is 0.2346 , the minimum value is 0.2339 , and the maximum value is 0.2356 . The average value of $y$ coordinates is 0.2754 , the minimum value is 0.2749 , and the maximum value is 0.2758 . The $x$ coordinate colour deviation is $0.001 . y$ coordinate colour deviation is 0.0005 .

The main luminescence of LED displays is the luminance of LED luminous tubes. At the same time, the main wavelength of the LED tube is related to the current of the LED display and the junc-

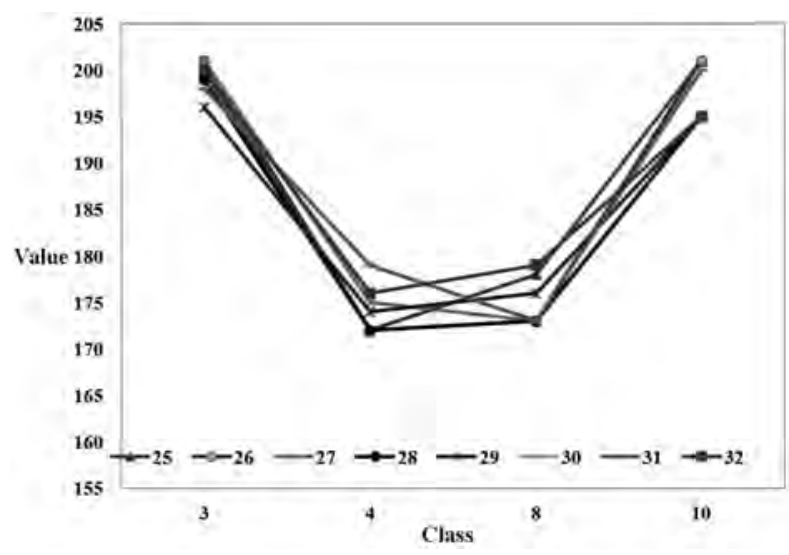

Fig.3. Gray-scale contrast between red super-light area between near area

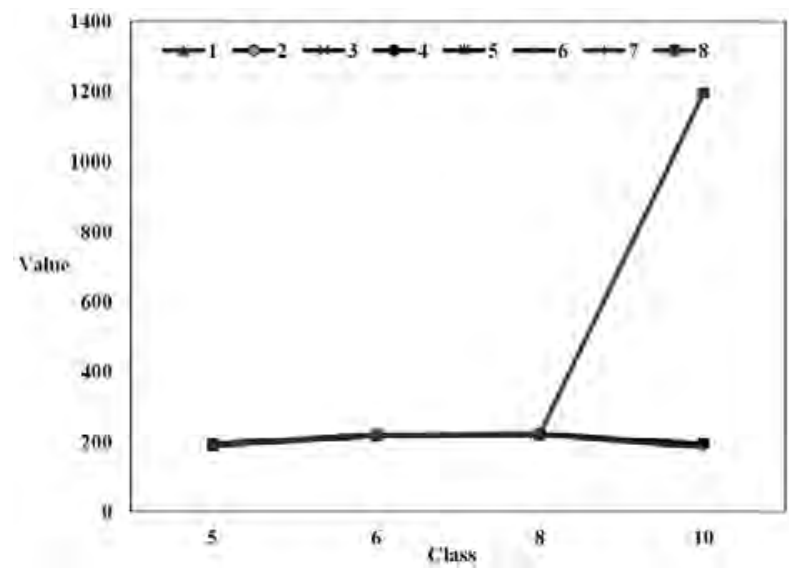

Fig.4. Gray-scale contrast between green dark-light area between near area

tion temperature difference of the semiconductor. The general LED luminous tube is tested at ambient temperature $25^{\circ} \mathrm{C}$ and working current $20 \mathrm{~mA}$. Environmental changes can give a certain impact on testing, so there will be some errors in on-site inspection. The display shows the function test results as shown in the Table 2.

After on-site monitoring, the brightness, contrast, brightness uniformity, white field chromaticity coordinates, display installation position and number finger, display control, display characters of Jingzhou Sports Culture Centre super large LED information release screen system meet the relevant requirements of GB/T 29458-2012 (requirements and test methods for LED display in Gymnasium).

A problem with the cell board, when the cell board displays red, there is a super bright problem in a certain area, the light in the red frame. When the unit panel displays green, there is a dark magic problem in a certain area, such as the lights 
Table 2. Display Non-Screen and Non-Functional Detection

\begin{tabular}{|l|l|}
\hline \multicolumn{1}{|c|}{ Project } & \multicolumn{1}{c|}{ Detection result } \\
\hline $\begin{array}{l}\text { Display position and } \\
\text { quantity of display } \\
\text { screen }\end{array}$ & $\begin{array}{l}\text { The site is set up with two flat LED displays, which are installed on the north and } \\
\text { south ends of the long axis of the field, and the two screens are all three base colours } \\
\text { (full colour).More than 95\% of the audience can meet the maximum sight distance. }\end{array}$ \\
\hline Display character & $\begin{array}{l}\text { The two LED displays on the site can display characters or Chinese characters on the } \\
\text { whole screen, and a small 16 dot matrix Chinese character is less than 12 lines, and } \\
\text { the distance between lines is not less than the character 1/1D.The association can dis- } \\
\text { play 36 Chinese characters. }\end{array}$ \\
\hline Display control & $\begin{array}{l}\text { Two LED displays can display the game time and the standard clock; it can display } \\
\text { the time of the game in real time and the result of the competition. The content of the } \\
\text { display can be manually switched; the video display screen, the text and picture, the } \\
\text { animation, the present live image can be automatically switched between each other } \\
\text { and handover manually. }\end{array}$ \\
\hline $\begin{array}{l}\text { Maximum sight distance } \\
\text { and character height }\end{array}$ & $\begin{array}{l}\text { The top two characters of the LED display are 0.38M and the maximum sight dis- } \\
\text { tance 131.1m. }\end{array}$ \\
\hline
\end{tabular}

in the green box. A dead light appeared when the cell board showed blue. The collection is red super bright area, green dark light area and adjacent area gray value.

Red, green, and blue question lights are marked respectively. Through Figs. 2-4 and combined with fault items, workers can quickly identify problems and make timely repairs. Since the centred coordinates of each lamp area correspond to the unit plate coordinates of a lamp, the function of automatically generating the plate coordinates of the wrong lamp point in the system is also set. On the one hand, the marks in the wrong pictures can help the workers to identify the wrong pictures quickly. The unit plate coordinates of the generated error points can be used to query and confirm the error problem. Because the following problems will directly cause a single pixel anomaly, multi pixel anomaly, single light often brightens, so the following problem is considered only when designing the wrong document. The document can be used as a reference document for maintenance, and the maintenance worker can quickly finish the maintenance after accurate positioning.

\section{CONCLUSION}

After ten years of development, China's industry has developed to a large scale. In the process of domestic industry construction, the standardization and standardization of products have long been highly concerned. The screen test method was officially introduced last year. In spite of the speci- fication of brightness in this document, there is no standardized facility to detect the differences in the brightness of each lamp in the display unit or by the way of personal work detection. The display unit detection system based on machine vision is introduced. In this system, the display unit pictures are taken by the camera, and each lamp in the display unit is collected by the digital image processing technology and the computer vision technology. And the lamp is determined according to the gray level criterion. The main contents are as follows:

- In order to carry out real-time and fast detection, the hardware circuit structure of real-time display and real-time shooting of display unit is designed;

- The choice of camera and lens is the most important part of the hardware device, which determines the quality of the picture taken by the camera. After comparing the parameters of each camera and lens, the camera and lens suitable for the system are selected;

- In order to reduce the noise pollution caused by the hardware and the external environment, the median filtering method is used to filter the salt and pepper noise in the pictures taken.

\section{REFERENCES}

1. Kozacki T, Chlipala M, Makowski P L. Color Fourier orthoscopic holography with laser capture and an LED display. Optics express, 2018. V26, \#9, pp.12144-12158.

2. Ahn H A, Hong S K, Kwon O K. An Active Matrix Micro-Pixelated LED Display Driver for High Lu- 
minance Uniformity Using Resistance Mismatch Compensation Method. IEEE Transactions on Circuits and Systems II: Express Briefs, 2018. V65, \#6, pp.724-728.

3. Kim D S, Im S K, Shigeta T, et al. High resolution LED display using a new rendering method with color sub-pixel architecture. Electronic Imaging, 2016, \#20, pp.1-4.

4. Xu, X., Xie, L., Li, H., \& Qin, L. Learning the route choice behavior of subway passengers from AFC data. Expert Systems with Applications, 2018. \#95, pp. 324-332.

5. Lv X, Loo K H, Lai Y M, et al. Energy-Saving Driver Design for Full-Color Large-Area LED Display Panel Systems. IEEE Trans. Industrial Electronics, 2014. V61, \#9, pp.4665-4673.
6. Zhang K, Peng D, Lau K M, et al. 25-5: Distinguished Student Paper: Fully-Integrated Active Matrix Programmable UV and Blue Micro-LED Display System-on-Panel (SoP). SID Symposium Digest of Technical Papers. 2017. V48, \#1, pp.357-361.

7. Hong Y, Lee B, Byun J, et al. 19-3: Invited Paper: Key Enabling Technology for Stretchable LED Display and Electronic System. SID Symposium Digest of Technical Papers. 2017. V48, \#1, pp.253-256.

8. Ejzak G A, Dickason J, Marks J A, et al. 512\$ times \$512, $100 \mathrm{~Hz}$ Mid-Wave Infrared LED Microdisplay System. Journal of Display Technology, 2016. V12, \#10, pp.1139-1144.
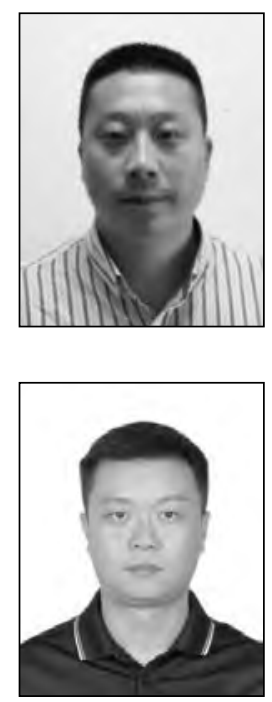

\section{Xiaodong YI,}

Master of Physical Education, Lecturer, Graduated from Sichuan University in 2009, working in Sichuan University, his interest include Sports and Physical Education

\section{Min ZHOU,}

Master of Physical Education, Lecturer, Graduated from Sichuan University in 2014, working in Sichuan University, his interest include Sports and Physical Education 